\title{
High-Fidelity Transmission Simulation for Hardware-in-the-Loop Applications
}

\author{
Orang Vahid \\ Paul Goossens \\ Maplesoft \\ 615 Kumpf Drive, Waterloo, ON, Canada, N2V 1K8 \\ ovahid@maplesoft.com pgoossens@maplesoft.com
}

\begin{abstract}
Model-based development plays a central part in optimizing existing transmission designs and exploring new system architectures. Design iterations and performance evaluations are done through virtual prototypes of the transmission systems, used in hardwarein-the-loop (HiL) simulations. In this paper, MapleSim's Driveline Component Library is introduced. The combination of this Modelica library and Maple's core symbolic technology, enables engineers to include more detail into their models targeted for real-time simulation of transmission systems. The paper also includes some results from the work at Aisin AW in modeling transmissions and HiL testing.
\end{abstract}

Keywords: Transmissions; Hardware-in-the-loop; symbolic calculations

\section{Introduction}

As automotive manufacturers strive to meet and exceed performance requirements on fuel efficiency and ride comfort, they have focused increasingly on the transmission design as one of the key factors. Engineers are putting tremendous effort into determining exactly how the power is lost, and what can be done to reduce losses and improve overall fuel efficiency. As a result, the transmission industry is now actively involved in optimizing existing transmission designs and exploring new system architectures. At the same time, transmission controllers are becoming more complicated and more detailed product testing is needed than ever before.

Model-based development (MBD) plays a central part towards achieving these goals. Design iterations are done through virtual prototypes of the transmission systems, used in hardware-in-the-loop (HiL) simulations. Virtual prototyping can yield more efficient products at significantly reduced costs by al- lowing engineers to address design issues long before they invest in physical prototypes.

In this paper we report on some of the activities under taken at AISIN AW in Japan regarding HiL simulation and the use of MapleSim environment to accelerate the development of automatic transmissions. The requirements for low calculation cost plant models for real-time simulations were met by creating the gear train part of the model in MapleSim. These models are then exported as optimized c-code for implementation into the HiL system.

\section{Transmission Modeling Using the Driveline Component Library}

The transmission models referred to in this paper are built using the components from the MapleSim Driveline Component Library (DCL) [1] as well as other components from the Standard Moldelica Libraries [2]. DCL covers all stages in a powertrain model from the engine through to the differential, wheels and road loads (See Figure 1). Furthermore, the library allows for flexible inclusion of power loss data that best reflect the way in which the loss data was acquired.

In the remainder of this section, some of the features of the components used in modeling transmissions are discussed.

\subsection{Clutches and Brakes}

As part of the standard component library, MapleSim provides two clutch models: a standard, controllable friction clutch and a one-way clutch [2].

In DCL, these models are expanded; clutch and brake models provide a real output port for the loss power and a Boolean output port to indicate clutch lock-up. There are also other formulation improvements that make DCL models perform better when used with fixed-step integrators usually encountered 
in real time applications and Hardware-in-the-Loop

tics. This is accommodated in the lookup table data simulations.

by providing torque ratios and capacity values for

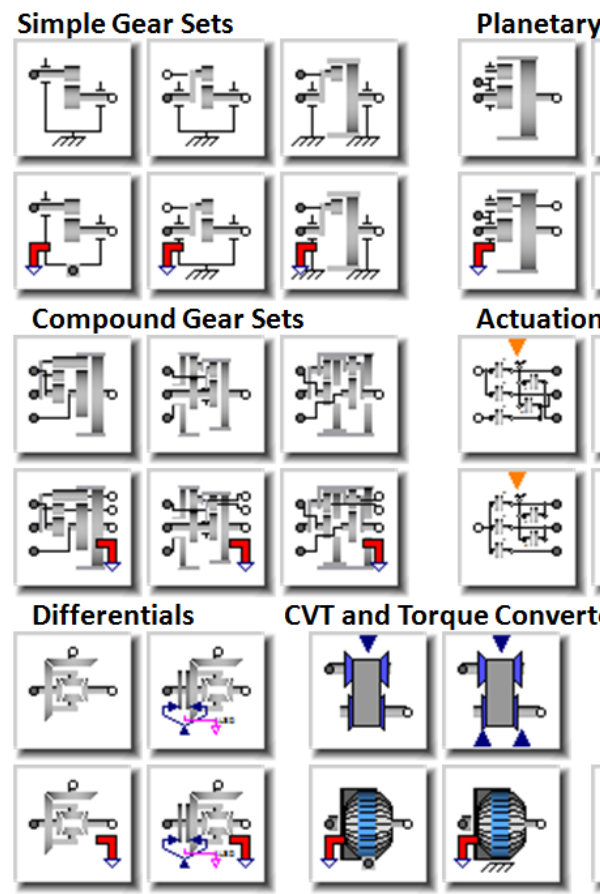

Figure 1: Driveline Component Library

\subsection{Torque Converter}

The torque converter is modeled using tables of measured data. The following characteristics are used:

- Torque Ratio $\frac{\tau_{t}}{\tau_{p}}$ vs Speed Ratio $\frac{\omega_{t}}{\omega_{p}}$

- Load Capacity $C$ vs Speed Ratio $\frac{\omega_{t}}{\omega_{p}}$

Where subscripts " $t$ " and " $p$ " designate turbine and pump quantities, respectively. The required data can be given as tabulated data. The Torque Converter component supports two alternative formulations based on the following definitions of the load capacity :

$$
\begin{aligned}
& \text { - } C=\frac{\tau_{p}}{\left(\omega_{p}\right)^{2}}\left[\frac{\mathrm{N} \cdot \mathrm{m}}{\mathrm{RPM}^{2}}\right] \\
& \text { - } C=\frac{\omega_{p}}{\sqrt{\tau_{p}}}\left[\frac{(\mathrm{rad} / \mathrm{s})^{1 / 2}}{\mathrm{~N} \cdot \mathrm{m}}\right]
\end{aligned}
$$

Backward flow, happens during deceleration of the vehicle where the vehicle kinetic energy is transmitted back through the transmission to the engine. In this situation, the turbine is pumping and the pump is acting as a turbine. Since torque converters are not designed to work optimally this way, the torque converter will have very different characteris-

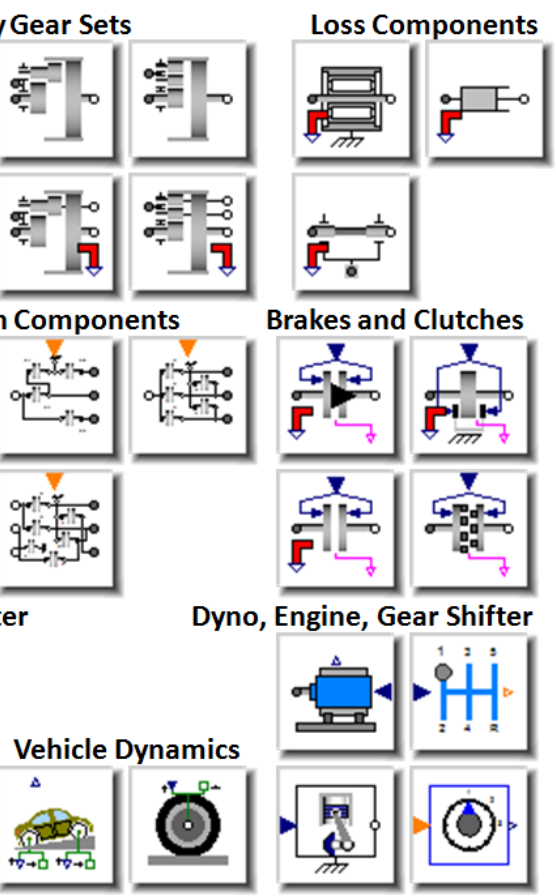

$\frac{\omega_{t}}{\omega_{p}}>1$, typically up to about 5 .

In the test model shown in Figure 2, the input (pump) torque is increased linearly for the first 10 seconds. At low speeds, between $t=0$ and $4 \mathrm{~s}$, the turbine torque increases faster than the input torque. This is the "torque multiplication" effect typically seen in the torque converters [3]. Due to the inherent inefficiencies in the mechanism, the turbine speed is slightly less than the pump speed while the torque is driving the pump.
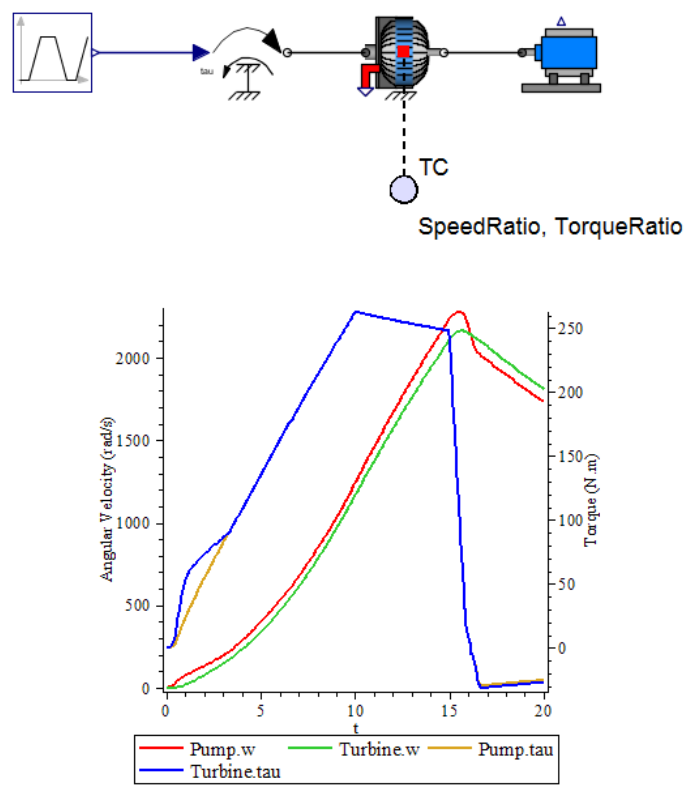

Figure 2: Torque converter test model 
Note that when the input torque drops at $\mathrm{t}=15 \mathrm{~s}$, the kinetic energy of the dynamometer changes the torque flow from forward to backward (i.e. turbine drives the pump), and the pump speed drops below the turbine speed.

\subsection{Gears, Gear Sets, and Transmissions}

DCL includes simple and compound gear sets and related actuation components for modeling gear trains and transmissions (see Figure 1). The Ravigneaux gear set component is discussed in the following as an example of the compound gear components in DCL.

\section{Ravigneaux Gear Set}

The Ravigneaux configuration is a basic automatic transmission planetary assembly. As shown Figure 3, this configuration is constructed internally using three Planet-Planet components and one Planet-Ring component.

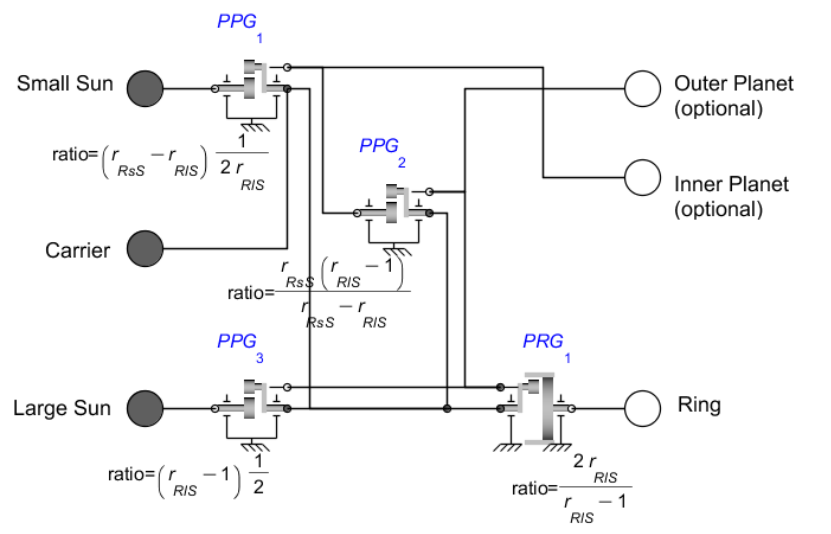

connect (PPG1.carrier, PRG1.carrier) ;

connect (PRG1. carrier, PPG2.carrier);

connect (PPG2 . carrier, PPG3. carrier) ;

connect (carrier, PRG1. carrier);

connect (small_sun, PPG1.inplanet);

connect (large sun, PPG3.inplanet): I

connect (PPG1 . outplanet, PPG2 . inplanet) :

connect (PPG2 . outplanet, PRG1 . planet);

connect (PPG2 . outplanet, PPG3. outplanet);

connect (ring, PRG1.ring) ;

connect (inner_planet, PPG1.outplanet);

connect (outer planet, PPG2. outplanet):

Figure 3: Internal structure of the Ravigneaux Gear

\section{Lepelletier Gear Sets}

There are two Lepelletier Actuation components (6speed and 7-speed) provided in DCL which can be used together with a Ravigneaux gear and a planetary gear to create 6-speed or 7-speed transmissions as shown in Figure 4-a and 4-b, respectively. (a)

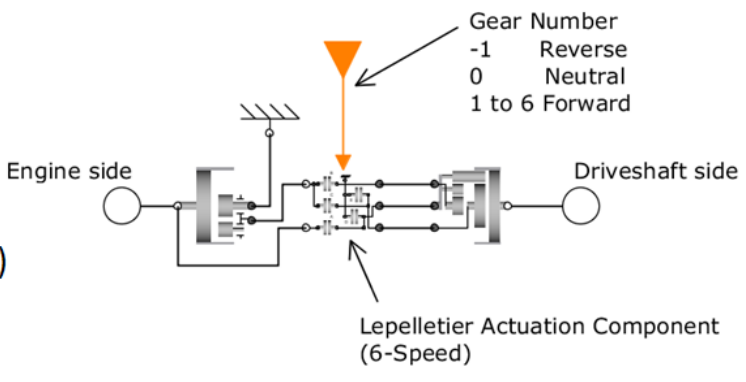

(b)

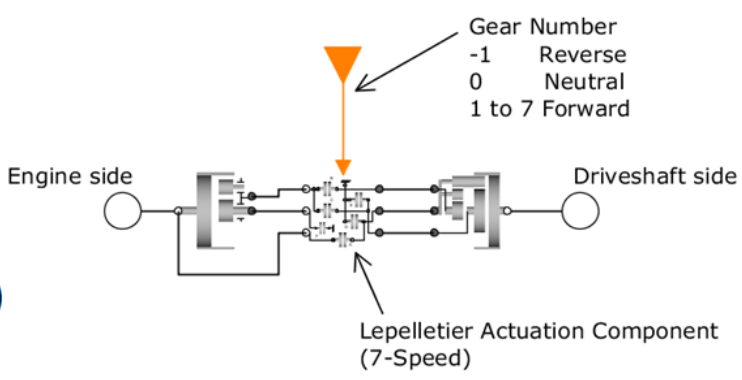

Figure 4: Building 6-speed (a) and 7-speed (b) transmissions with the Lepelletier Actuation components

\subsection{Incorporating Losses}

As shown in Figure 5, all of the gear components in the DCL can easily be switched from ideal (i.e. no losses) to lossy where power losses due to toothmeshing are accounted for [4].

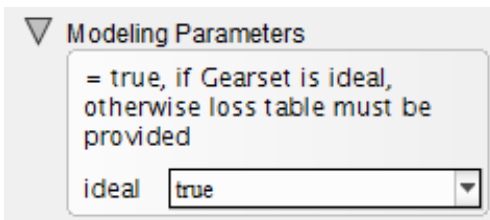

\section{Figure 5: Fundamental GUI option for all gears - ideal $=$ true/false}

In lossy mode, the meshing friction is expressed as a transmission efficiency $\left(\eta(\omega)=\tau_{\text {out }} / \tau_{\text {in }}\right)$ which may be defined as a function of the gear angular velocity via data tables. The user has the option to provide an efficiency table for each meshing gear pair in the gear set individually.

In compound gear sets (Planetary Gear, Dualratio Planetary Gear, Counter-rotating Planetary Gear, Ravigneaux Gear, Simpson Gear, and CR-CR Gear), internal bearing damping can be added using the component options. Bearing friction can also be added using external Bearing Friction components by enabling the optional "planet flanges". Figure 6 shows a Counter-rotating Planetary Gear component with added bearing friction losses. The bearing friction is expressed as a torque loss and is related only to the shaft speed [2]. 


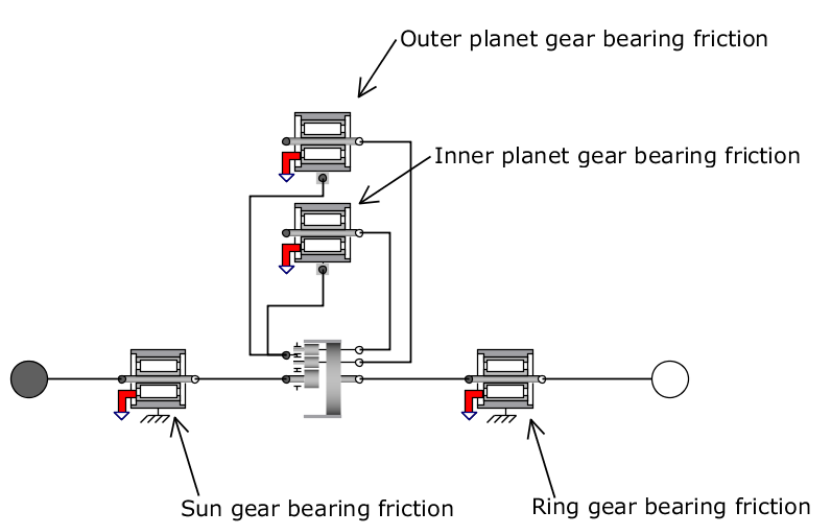

Figure 6: Adding bearing friction to gear sets

\section{Advantage of the Symbolic Tech- nology}

Symbolic techniques turn out to be a critical ingredient, both to enable efficient modeling of these components as well as to generate optimized code, yielding the required HiL execution speed. MapleSim's symbolic capabilities are enabled by an underlying Maple computation engine [6], providing extremely efficient symbolic operations that are necessary for handling the thousands of system equations typically found in the transmission models.

A common characteristic of Modelica environments is that system models are built by assembling components using "physical" connections, carrying quantities like torque and rotational angle bidirectionally between the two components. The decision on causality of the model is deferred to simulation time, just before the numeric integration process is started. This is possible because the entire set of equations for the whole system is generated symbolically, as a first step. At this point we typically have a set of differential algebraic equations. As shown in Figure 7 , several steps are necessary before these equations can be solved numerically, yielding simulation results and/or HIL code. These steps are discussed next.

\subsection{Equation Simplification}

The initial set of equations generated from the system model is typically large and contains many redundancies. Symbolic techniques are used to simplify this set of equations as much as possible. The simplifications are exact and do not result in any loss of fidelity in the model. Trivial equations of the form $a=b$ are removed. Linear equations are pre-solved analytically. Reducing the number of equations by a factor of ten is not uncommon. This simplification step is key to the scalability of the remaining preprocessing steps.

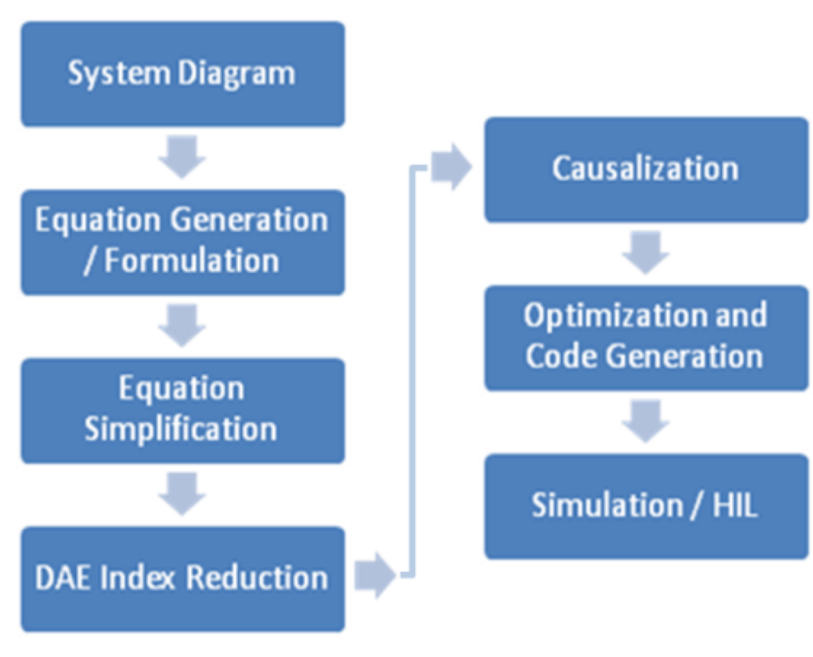

Figure 7: Steps towards numerical simulation

\subsection{Index Reduction}

The generated system consists of differential algebraic equations (DAEs). Such equations cannot be readily solved with standard numerical techniques because of the presence of algebraic constraints. The "index" of a DAE is loosely defined as the number of times the equations need to be differentiated in order to remove these constraints. The goal here is to reduce the system of equations to "index 1", allowing numeric integration. During integration, the constraints are monitored for "drift", ensuring an accurate solution, reflecting the behaviour of both the differential equations as well as algebraic constraints. Again, symbolic techniques turn out to be essential, allowing differentiation of equations and efficient index reduction.

\subsection{Causalization}

At this point, we have a simplified system of (index 1) differential equations. In order to numerically solve this system, we will need to repeatedly evaluate the system for a particular point. To enable this, we will need to turn our (acausal) system of equations into a (causal) sequence of numeric operations. In short, this process involves imposing an order of evaluation onto our set of equations. Doing this efficiently involves tools from graph theory, readily available in the symbolic computing tool chest.

\subsection{Optimized Code Generation}

Executing speed is critical to HiL applications and symbolic techniques again turn out to be key to gen- 
erating highly efficient code. It is, of course, possible to generate code directly from the causal system of equations described above. However by looking at those equations globally, we are able to perform symbolic optimizations prior to generating code, which makes the difference between achieving the required HiL cycle times or not. These optimizations involve detecting common computation sequences that can be factored out, which go way beyond the (local) optimization capabilities of available compilers.

\subsection{Two Examples}

\section{A Simple Driveline Model:}

Consider the driveline mode shown in Figure 8. The model represents a vehicle powertrain from engine to dynamometer. The model includes a torque converter between the flywheel and the transmission. The transmission is a 4-speed Ravigneaux gearbox. Using throttle and brake controllers, the speed is changed following a ramp-up/coast down profile.

Using MapleSim's API commands from Maple, the simulation time is measured. A fixed time-step solver (Euler) is used here with a time step of 0.001 sec. Total simulation time is 150 seconds. The simulation was done on a 64-bit Windows 7 machine with Intel(R) Core(TM) Duo $2.40 \mathrm{GHz}$ CPU. Figure 9 shows Maple's commands for this example. These command extract and simplify the model equationsand convert them to optimized c-code. The simulation results are obtained from a Maple procedure which includes the complied c-code.
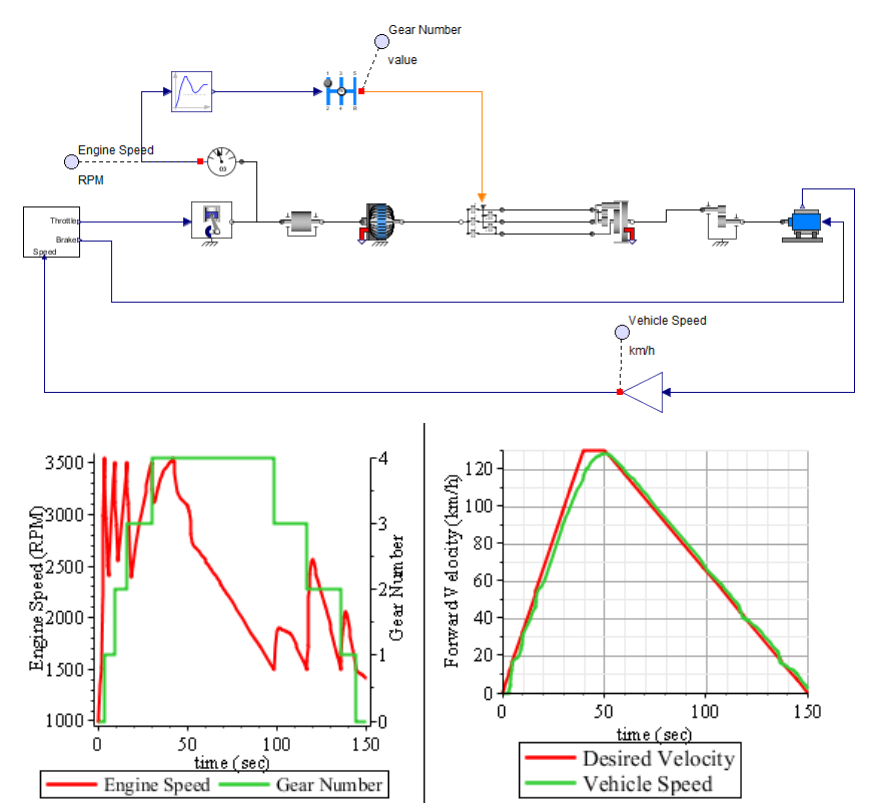

Figure 8: An example of a complete powertrain.
The simulation was done over 15 times faster than real-time (i.e. $\sim 10$ second of integration time for a 150 -second simulation). In 20 consecutive runs the average simulation time was 9.68 with standard deviation of 0.30 .

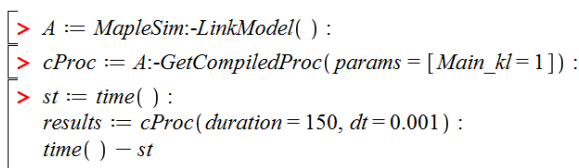

\section{Figure 9: Running MapleSim simulation using} API commands in Maple

A Vehicle Model with Mean-value Engine Model: The system in Figure 10, is the second example chosen for the real-time demonstration. This model is considerably more complex than the previous example and includes a detailed mean-value engine model [7] and a 4-speed transmission model. The MapleSim model uses the New York City Cycle [8] and runs for 600 seconds. Simulation timing was done under similar solver settings as the previous example. The same computer was also used. On average the simulation was done about 12 times faster than real-time (i.e. $\sim 50$ second of integration time for a 600-second simulation). Based on 15 consecutive runs the average simulation time was 50.2 seconds with standard deviation of 0.54 .

\section{HiL Simulation of the Automatic Transmissions}

At AISIN AW, HiL simulation is extensively used to accelerate the development of automatic transmissions. The plant models for HiL simulations require sufficiently high fidelity to accurately represent the aspect of the system dynamics important to the designers. At the same time, these models have to have low calculation cost in order to enable real-time execution.

As shown in Figure 11, the real-time platform used in the HiL simulations reported here is the ADX system [9] from A\&D Technology, Inc.

The plant model is deployed in Simulink [10] and can be separated into two parts as depicted in Figure 12. The first part is the plant model which is constructed of the s-function generated from MapleSim models including clutches, brakes, and various gear sets. This part also includes Simulink blocks for other parts of plant model. The second part is the automatic testing module.

It is critical that the calculation time associated with the first part (plant model) is kept as low as 


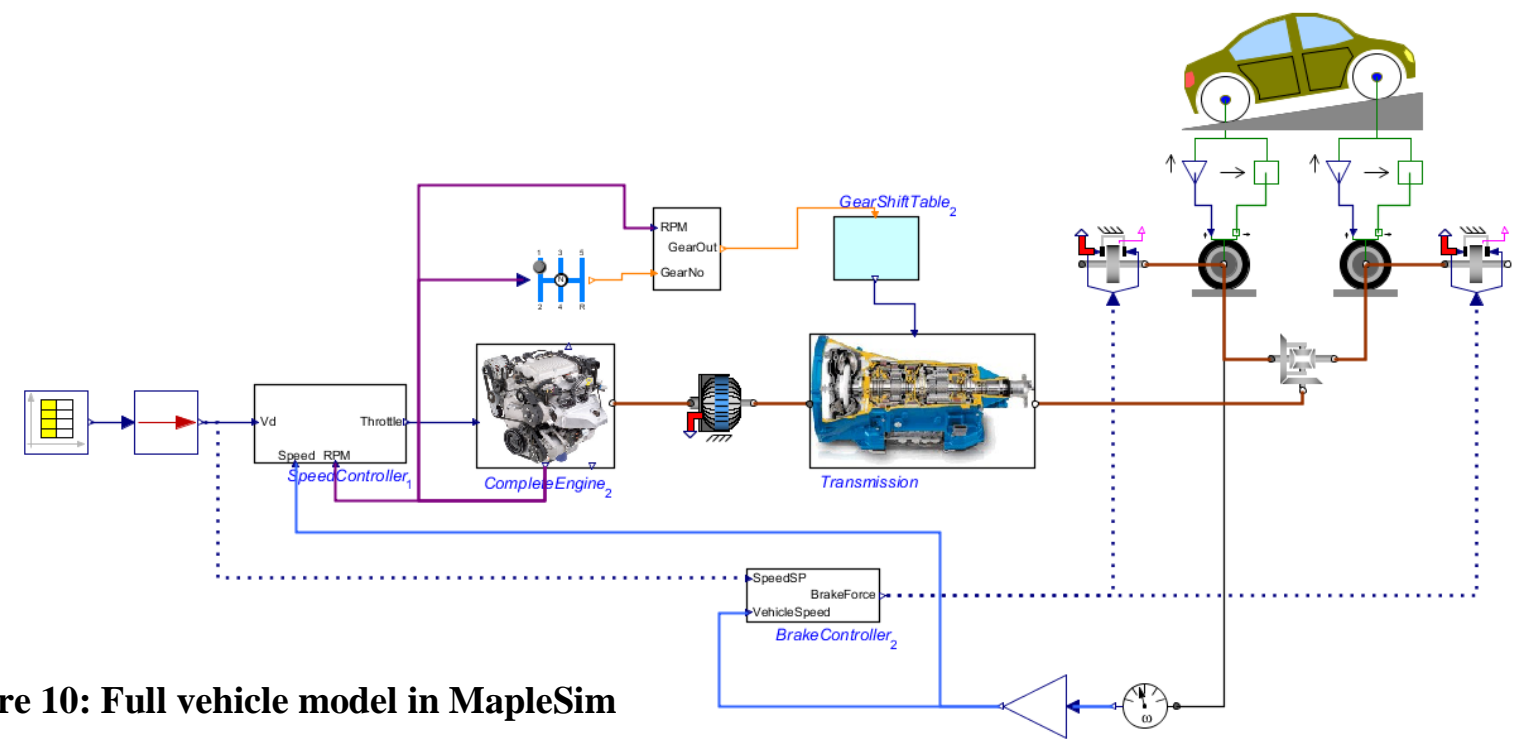

possible to accommodate for the high execution times of the increasingly more complex automatic testing routines implemented in the testing module.

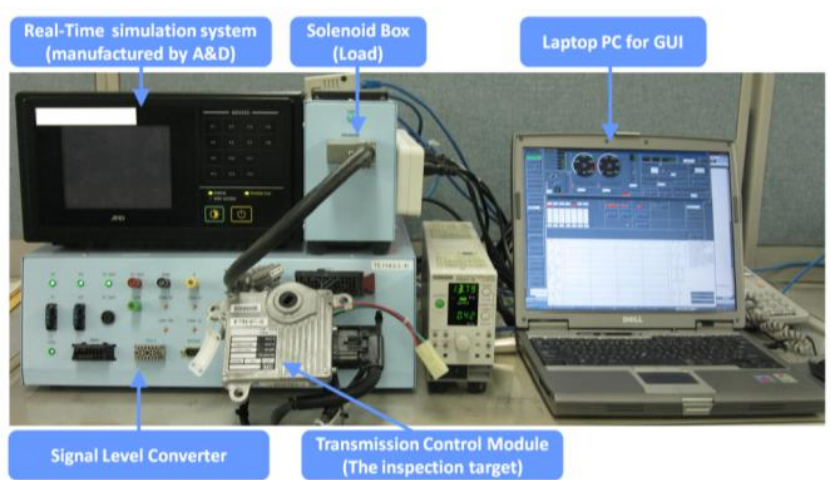

Figure 11: HiL simulation system
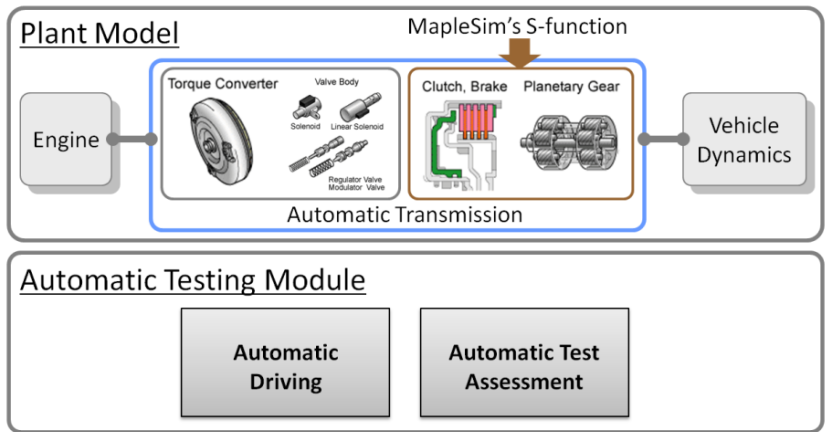

Figure 12: Model for Real-time system

A sample gear train is shown in Figure 13 which includes a planetary gear, a Ravigneaux gear, and a basic gear connected together using three clutches, two brakes (modeled using clutch components), and a one-way clutch. This gear train is connected to an ideal gear which represents the differential gear ra- tio. The tire load is modeled using additional inertia, clutch, and brake components. The tire component and the longitudinal vehicle dynamics component (refer to Figure 1) are not used here since that level of fidelity is not necessary for the intended HiL simulations.

Figure 14 shows the HiL simulation results with s-function generated from MapleSim. Compared with another software previously used, it was shown that for the above model, the implementation of the s-function generated from MapleSim in the HiL simulations with a sampling time of $1 \mathrm{~ms}$, reduced the overall CPU time by $250 \mu$ s (or $25 \%$ of a time step). This reduction is due to fact that the MapleSim's sfunction runs twice as fast as the previously implemented block.

\section{Conclusions}

In this paper some of the features of the Driveline Component Library - an add-on Modelica library for MapleSim modeling, simulation, and analysis environment - were introduced. The Driveline Component Library provides a comprehensive set of components that enable transmission manufacturers - as well as other automotive developers - to conveniently create plant models for control and simulation. The underlying symbolic computation engine of MapleSim (i.e. Maple) expands the inherent advantages of similar Modelica-based physical modeling tools to new heights. Benefiting from the power of symbolic computing, MapleSim can generate extremely fast code that is of vital importance when simulating large complex systems in real-time. 


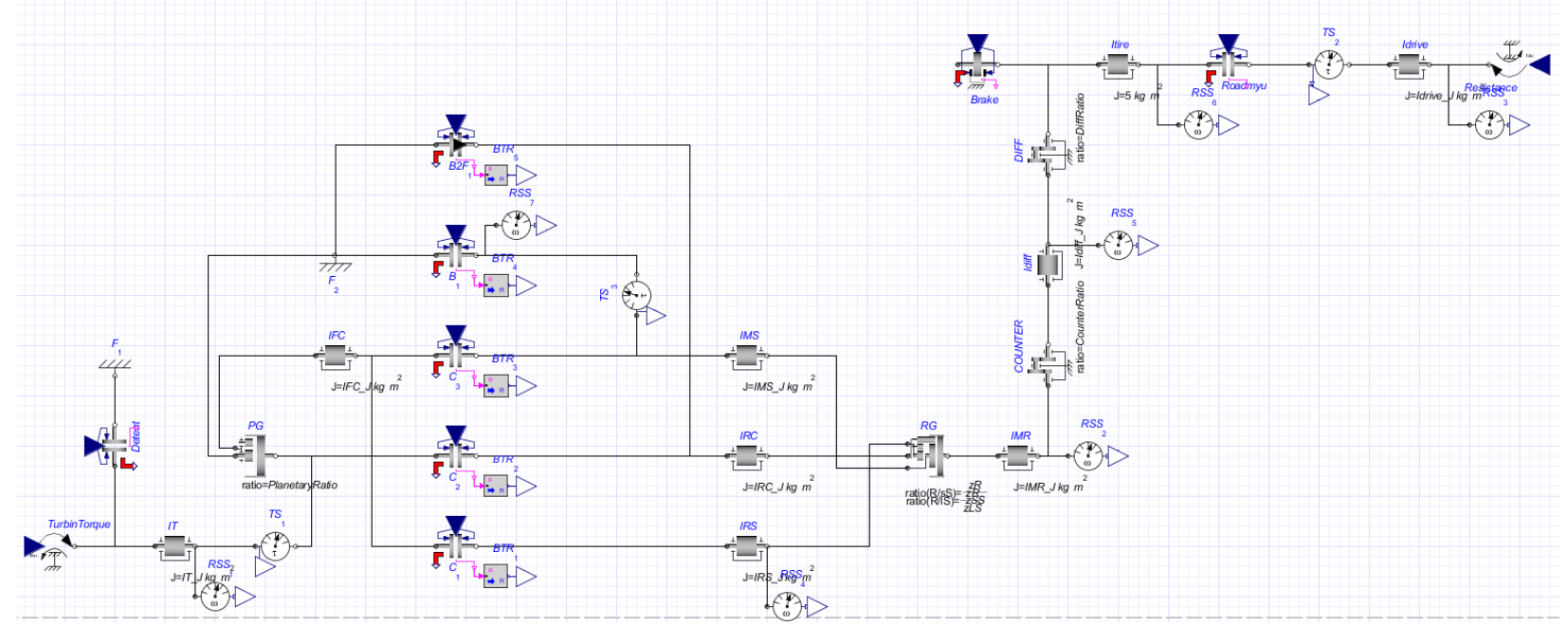

Figure 13: Gear train model created in MapleSim

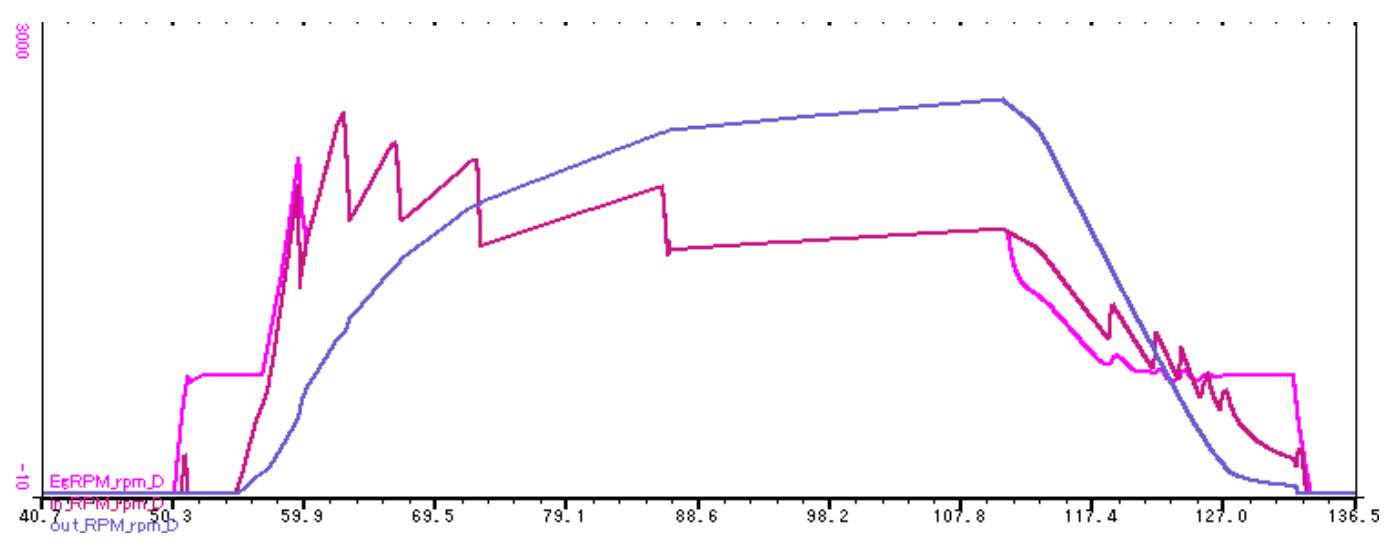

Figure 14: A sample of HIL simulation results

The paper also included a brief description of the activities at AISIN AW on the development of new automatic transmissions and their use of MapleSim and the Driveline Component Library in HiL simulations. The optimized c-code generated by MapleSim from transmission plant models enabled AISIN AW to perform more detailed HiL simulations. In a sample case study, it was shown that the s-function generated by MapleSim ran twice as fast as the sfunction generated by a similar tool.

\section{References}

[1] MapleSim User's Guide, 2011, ISBN 978-1926902-09-8.

[2] https://www.modelica.org/ (accessed 2/4/2012).

[3] D. Hrovat and W.E. Tobler. "Bond graph modeling and computer simulation of automotive torque converters," Journal of the Franklin Institute. Volume 319, Issues 1-2, January-February 1985, pp 93-114.
[4] Pelchen C., Schweiger C., and Otter M., "Modeling and Simulating the Efficiency of Gearboxes and of Planetary Gearboxes," $2^{\text {nd }}$ International Modelica Conference, Proceedings, pp. 257-266.

[5] Joško Deur, Vladimir Ivanovic', Matthew Hancock, and Francis Assadian. "Modeling and Analysis of Active Differential Dynamics," Journal of Dynamic Systems, Measurement, and Control, 2010. Volume 132 / 061501, pp 1-14.

[6] Bernadin L., Chin P., DeMarco P., Geddes K. O., Hare D. E. G., Heal K. M., Labahn G., May J. P., McCarron, Monagan M. B., Ohashi D., and Vorkoetter S. M., Maple Programming Guide, 2011, ISBN 1-92690208-1.

[7] - , "Mean-Value Internal Combustion Engine Model", Maplesoft, White Paper, http://www.maplesoft.com/contact/webforms /whitepapers/enginemodel.aspx, (accessed: 2/4/2012). 
[8] -, Dynamometer Driver's Aid, http://www.epa.gov/nvfel/testing/dynamomet er.htm, (accessed: 2/4/2012).

[9] http://www.aanddtech.com/ADX.html (accessed: 2/4/2012).

[10] http://www.mathworks.com/products/simulin k/ (accessed: 2/4/2012). 